\title{
Éloge de la folie ou folie de l'éloge
}

\section{Henri Suhamy}

\section{(2) OpenEdition \\ Journals}

Édition électronique

URL : http://journals.openedition.org/shakespeare/1307

DOI : 10.4000/shakespeare.1307

ISSN : 2271-6424

Éditeur

Société Française Shakespeare

Édition imprimée

Date de publication : 1 novembre 1989

Pagination : 9-16

Référence électronique

Henri Suhamy, «Éloge de la folie ou folie de l'éloge », Actes des congrès de la Société française

Shakespeare [En ligne], 7| 1989, mis en ligne le 01 janvier 2007, consulté le 04 mai 2019. URL : http:// journals.openedition.org/shakespeare/1307 ; DOI : 10.4000/shakespeare.1307 


\title{
Société Française Shakespeare
}

\author{
Actes des Congrès \\ 1985 - 1986 - 1987
}

\section{LA FOLIE}

\section{SHAKESPEARE ET LES ARTS \\ LE TRAGIQUE}




\title{
ELOGE DE LA FOLIE OU FOLIE DE L'ELOGE?
}

\author{
Henry SUHAMY
}

\begin{abstract}
Une question se pose à chacun de nous : entre la Raison et la Folie, que choisir?

Le public pourrait cependant objecter qu'une question posée de façon si sommaire se heurte d'abord à la difficulté de définir les termes. Et ensuite qu'il y a peut-être un malentendu sur le sujet à traiter, car nous ne sommes pas réunis ici pour débattre d'un dilemme frivole ayant trait à la vie quotidienne, mais pour parler de choses sérieuses, de littérature, de théâtre, de Shakespeare.

Je demande cependant la permission de m'obstiner dans cette voie, qui vient d'un certain souci de rationalisme. En tant que cartésien - car on a le privilège en France de pouvoir se proclamer cartésien sans même avoir lu Descartes - j'ai éprouvé, devant le programme général de ce colloque, une double crainte, sans doute injustifiée, sans doute inspirée par ma mauvaise foi et mon mauvais esprit habituels. La crainte d'abord d'entendre pendant deux jours un éloge nostalgique et systématique de la folie, présentée comme la source de toutes les libertés, de toutes les inventions, de toutes les intuitions, quitte à ce que, une fois le colloque terminé, le rideau tombé sur le carnaval oratoire et ces savantes saturnales, chacun rentre tranquillement chez soi et reprenne le cours d'une vie fondée sur la raison, ou ce qui en tient lieu, c'est-à-dire la prudence, la sagesse mondaine, le conformisme, la respectabilité, le souci de la carrière universitairre. L'imagination, prudemment historicisée et artistiquement sublimée, n'aura donc pris le pouvoir que par interim. Pourquoi alors ne pas faire plutôt l'Eloge de la Raison? A cette crainte s'en ajoutait une autre, celle d'entendre attribuer à Shakespeare le même genre de complaisance envers la folie sous toutes ses formes, car il existe une longue tradition en France qui veut qu'à chaque fois qu'on parle de Shakespeare on décrète le tumulte et on ferme le temple de Janus, ce qui occulte la deuxième face de ce Janus, la face rationaliste. Il faut toutefois reconnaître que cet éloge de la folie s'appuie sur un dossier
\end{abstract}


considérable. Il n'y a pas si longtemps que le philosophe Michel Foucault, dans son Histoire de lafolie a l' âge classique, présentait la Renaissance comme l'Age d'or de la folie, et soutenait la thèse selon laquelle la notion même de folie pathologique fut inventée par le rationalisme classique comme une sorte de repoussoir. Foucault va même jusqu'à laisser entendre que c'est en partie pour utiliser les hôpitaux désaffectés et désinfectés à la suite de l'extinction des grandes épidémies de lèpre et de peste que la Société décida d'interner les malades mentaux. Il y a peut-être une part de vérité dans ces théories, mais nous savons en tant qu'anglicistes, que le 16e siècle traitait parfois les déments de façon barbare et culpabilisante, et que les épidémies de peste n'ont pas empêché Bedlam de prospérer.

Pour revenir maintenant au point de départ, c'est-à-dire la question de savoir s'il est moralement et philosophiquement légitime de faire l'éloge de la folie sans tomber dans un discours de dilettante ou d'idéologue, nous retrouvons la difficulté préalable qui vient de ce que les notions elles-mêmes que nous sommes amenés à manipuler ont des contours et des contenus indécis. D'autre part nous étudions ici la littérature anglaise, en particulier Shakespeare. Or nous savons combien il est difficile de traduire des mots comme fool et folly. En vérité ces obstacles linguistiques ont quelque chose d'utile et de salutaire. Ils nous avertissent de l'ambiguîté subtile et glissante de ces concepts, si chargés d'autre part de connotations éthiques et affectives. Si l'on applique la méthode mécanique du calque, et qu'on traduise fool et folly par fou et folie, on se trompe à peu près une fois sur deux, car les termes anglais en question dénotent en principe la sottise, la balourdise, l'ignorance, la stupidité, et non l'état de démence, qui correspond plutôt au mot madness. Mais il y a de nombreux cas où le texte et le contexte justifient la traduction parfou et folie, car en anglais folly et madness ont des sèmes communs, de même que sottise et folie en français, qui sont censés avoir des référents dans la réalité psychologique. Il n'est pas inutile de rappeler que symétriquement raison et sagesse ont le même genre de contiguitté. Transposées sur la scène et stylisées par convention artistique, les diverses facettes de la folly - je suis obligé de recourir au mot anglais - produisent des emplois de thêâtre bien connus, le plus souvent comiques, quelquefois pathétiques, tels que le bouffon, le simple d'esprit, le rustre, le fou de cour, entre lesquels il existe des différences, mais également des zones communes, de même qu'entre l'ensemble de ces divers types comiques et le dément au sens pathologique. Comme l'a très bien démontré Victor Bourgy dans son ouvrage classique sur la question, ces personnages présentent un arc-en-ciel de nuances distinctes, mais capables de se fondre les unes dans les autres.

Les bouffons et les fous signalés comme tels ont un caractère commun et significatif : 1 'absence de sagesse mondaine et de réflexes d'obéissance, 1 'insoumission aux convenances sociales. Le simple d'esprit s'appelle parfois le naturel. En termes freudiens on dirait qu'ils n'ont pas de surmoi, ils ne connaissent pas de censure, intérieure ou extérieure.

Quant à la nécessité d'établir des distinctions entre les diverses formes de sottise ou de folie fonctionnelle, elle ne répond pas seulement au souci légitime de 
discernement intellectuel, mais aussi à une exigence morale. S'il existe une folie digne d'éloge, laquelle?

Si l'on s'appuie sur le thêâtre de Shakespeare, il apparaît clairement que toutes les folies ne sont pas dignes d'éloge, car s'il est vrai que cette œuvre ne contient guère de sermons au premier degré, du moins adressés directement au public, et qu'il y règne un esprit de tolérance et d'objectivité que l'on a pris quelquefois pour du scepticisme intégral, elle sollicite toujours d'une manière ou d'une autre la réflexion morale du spectateur. Certains des bouffons shakespeariens n'attirent guère la sympathie, et par contre-coup le type d'irresponsabilité qu'ils représentent n'a pas de valeur exemplaire. Heureux de constater qu'on les a une fois pour toutes catalogués comme individus asociaux, ils versent volontiers dans le cynisme et le parasitisme, l'irrespect déplacé. La distance qui sépare fool et knave, c'est-à-dire sot et coquin, n'est pas toujours très grande. Mais certains d'entre eux, notamment le plus célèbre, le Fool anonyme du Roi Lear, possèdent ou cultivent une candeur transparente, une innocence radicale, qui leur conferent un statut quasi évangélique. Ses nombreux fioretti en font le Saint François du thêatre shakespearien.

Voici par exemple comment il s'étonne que Kent veuille servir le vieux roi, et comment il explique en substance pourquoi il lui est resté fidèle. Mon maître a perdu tout son pouvoir, donc tout le monde l'abandonne, conformément aux normes de la sagesse d'ici-bas. Si je lui reste loyal, c'est parce que je suis fou. Ou inversement, si on me considère comme fou, $c$ 'est parce que je lui reste loyal. Les deux conclusions se valent. Et le Fou pousse la logique de son raisonnement, ou antiraisonnement jusqu'à rester fidèle à Cordelia devant Lear de même qu'il reste fidèle à Lear devant les deux autres filles. On voit que cette sottise très élaborée constitue un ensemble de procédés rhétoriques fondés sur l'ironie. La leçon est claire. Puisque nous vivons dans un monde qui ne croitplus aux valeurs de la chevalerie - rappelons au passage que Le Roi Lear est exactement contemporain de la première partie de Don Quichotte - puisque l'instinct de conservation et la volonté de puissance sont devenus les seules normes, quiconque ne se conforme pas à ces exigences machiavéliennes doit demander pardon, plaider coupable, invoquer comme excuse l'irresponsabilité mentale. Bien entendu, nous sommes ici au thêtre, comme dans la scène de Macbeth où Lady Macduff tient des propos analogues, bien qu'elle ne donne aucun signe de faiblesse mentale ou de chavirement.

Une telle virtuosité dans le discours satirique relève de l'exploitation littéraire du thème bien connu de l'innocent, et non de la description psychologique de la simplesse ou de l'aliénation.

On comprend pourquoi un écrivain peut se permettre de confondre candeur et folie, pourquoi il lui est si facile de passer de la stupidité à la démence et de la démence à l'illumination, puisqu'il s'agit dans beaucoup de cas de la production d'un discours qui signale clairement son appartenance au délire dénonciateur, et qui fabrique son propre support. On sait qu'à partir du moment où Lear est frappé de démence, il se metà tenir des propos nihilistes semblables parfois à ceux que débitait 
son bouffon jusque-là. Comme ce dernier disparaît de la scène au troisième acte, on a souvent dit que Lear devenait son propre fou, et que le précédent $n$ 'avait donc plus de raison d'être, ou de folie d'être, d'où son départ. Mais cela entraîne qu'il n'y a pas de différence de nature entre l'innocence congénitale de l'un et la démence pathologique et accidentelle de l'autre. Tout serait donc une affaire de discours et de convention littéraire. La folie n'aurait plus aucun poids psychologique ou existentiel. Elle fonctionnerait seulement comme un procédé satirique destiné à renvoyer à la sagesse mondaine sa caricature et sa condamnation. Alors l'éloge de la folie va de soi s'il s'agit d'un procédé destiné à blâmer tout ce qui est blâmable, un petit garçon d'Andersen aux yeux éternellement lucides et au franc-parler éternellement candide.

Cette conception de la folie comme outil satirique manque peut-être un peu de substance humaine, mais elle a des états de service très respectables, liés notamment à un renouveau de l'esprit évangélique.

Tout le monde sait que l'Eloge de la Folie d'Erasme s'est d'abord intitulé Encomium Moriae pour deux motifs importants. Moria est un mot grec, transposé en latin, qu'Erasme a choisi pour en faire un jeu de motsà usage privé. Le dédicataire de l'ouvrage n'était autre que Sir Thomas More, alias Thomas Morus ; or morus est la transcription latine du grec mwros, adjectif qui correspond à mwria, la sottise. Certes, Erasme dit dans sa dédicace que c'est par antiphrase que Thomas Morus, le plus sage des hommes, porte un tel nom. Mais il laisse entendre aussi que la sagesse de Thomas More n'est pas du genre de celle que les hommes reconnaissaient habituellement comme telle. Grand humaniste, adversaire de la scolastique, pratiquant de ce piétisme candide qu'on appela la Nouvelle Dévotion, homme naturellement bon et spontané, jovial et généreux, Sir Thomas More incarne cette candeur ingénue qui peut passer pour une forme de folie, de folie souriante et idéalisée.

N'est-il pas émouvant de songer que Sir Thomas More fut victime en quelque sorte de sa propre moria, c'est-à-dire de sa loyauté envers l'Eglise catholique qu'il avait critiquée autant que le Fou de Lear critique son maître, mais à laquelle, bien que laïc, il resta indéfectiblement fidèle ? Bel exemple de noble folie poussée jusqu'au sacrifice. Folie béatifiée, sinon béate. Et il n'est pas indifférent que le dédicataire de l'Encomium Moriae soit allé jusqu'au bout de son engagement religieux, car la Moria en question a pour Erasme une signification essentiellement chrétienne. Au centre de l'ouvrage se trouve un verset de la première Epître aux Corinthiens (I.18.21) où Saint Paul, citant Isaïe, écrit ceci :

Pour ceux qui périssent, la parole de la croix est folie (mwria dans le texte grec) mais pour nous qui sommes sauvés, elle est la puissance de Dieu. Car il est écrit :

Je détruirai la sagesse des sages,

Et $j$ 'anéantirai l' intelligence des intelligents.

Où est le sage? Où est le docte ? Où est le dialecticien de 
ce temps? Dieun'a-t-il pas convaincu de folie la sagesse du monde? Car puisque le monde en sa sagesse n'a pas reconnu Dieu dans la sagesse de Dieu, il a plu à Dieu de recourir à la folie de la prédication pour sauver les croyants. dans la phrase

L'original grec contient donc le mot mwría ainsi que le verbe mwraiveiv

ouci emwranen o qeos thu sojiau tou kosmou

que l'on traduit quelquefois par «Dieu n'a-t-il pas rendu folle la sagesse du monde ? , mais dont le sens est peut-être mieux exprimé par la traduction que j'ai empruntée à Louis Segond, «convaincu de folie». L'idée, toutefois, de la folie infligée aux hommes comme un châtiment ne manque pas d'intérêt, etl'on pense aux transes douloureuses dont Ariel et Prospero punissent leurs ennemis, mais n'anticipons pas. Et puisqu'il est question encore une fois de traduction, on peut rappeler que dans la vulgate, Saint Jérôme a traduit mwría par stultitia et mwraivev par stultam fecit, ce qui n'entraîne aucune surévaluation du concept. On sait aussi que le second titre choisi par Erasme est précisément Laus Stultitiae, que l'on aurait le droit de traduire par Eloge de la Stupidité. Et pourtant la traduction habituelle se justifie. Les mots sottise ou stupidité n'ont pas les mêmes résonances, le même prestige, même dans la négativité, que le mot folie. A vrai dire il y a un danger de cercle vicieux, car si à force d'accumuler des connotations laudatives, la notion de folie en arrive à inclure son propre éloge, l'acuité paradoxale du titre d'Erasme finit par s'émousser, ainsi que l'ironie et le balancement inconfortable qui va constamment de l'éloge à la moquerie. Mais il y a autre chose. Ce n'est pas seulement pour sa beauté ambiguë que l'on peut conserver le titre traditionnel. L'ensemble du texte le justifie. La folie selon Erasme, - compatriote de Breughel, fils naturel du pays des kermesses, - ce n'est pas seulement la candeur puérile et prophétique qui va tout droit à la vérité de Dieu en évitant le piège de la sagesse mondaine ou le labyrinthe des ratiocinations pharisiennes et scolastiques. C'est aussi le grand jaillissement de la Création, le rire de la Nature, le désordre burlesque de la vie. Et dans sa prosopopée, Moria se désigne parfois sous des appellations quiévoquentla démence pathologique : amentia, c'està-dire l'absence de facultés mentales : dementia, la perte de l'esprit ; insania, la maladie, avec la spécialisation que l'on sait.

Ne nous attardons pas sur l'ironie du texte qui joue constamment sur l'interchangeabilité de folie et sagesse. La Folie ne fait pas seulement son propre éloge, elle loue également ceux qu'elle appelle ses disciples, et qui n'ont généralement pas lieu de se sentir flattés. Toutcela fut résumé par Montaigne dans sa fameuse phrase, «Ce n' est pas la folie des hommes qui me fait rire, c' est leur sagesse» ou par le cas très particulier de Hamlet qui trouve l'ironie suprême de se faire passer pour fou, y compris auprès de certains lecteurs, en disant des choses en somme très 
raisonnables.

Ce qui est intéressant dans la confusion volontaire entre Stultitia et dementia, pour nous qui nous intéressons à Shakespeare, c'est qu'elles produisent à peu près le même discours. Et que ce discours a un contenu spirituel. Dans Shakespeare l'innocence mentale qui correspond au mot folly a toujours un caractère congénital, tandis que la démence qui correspond à madness est toujours une maladie accidentelle, due à des causes psychosomatiques précises. Mais on a vu que les discours produits par ces deux formes d'aliénation se ressemblent, et que dans Lear, le roi lui-même se lance dans une contestation sauvage qui rappelle la désespérance radicale de son bouffon, tandis qu'Edgar dans sa simulation, tombe dans une hallucination visionnaire, qui une fois déchiffrée, constitue un véritable sermon. Il joue le rôle d'un pécheur qui souffre d'une psychose de remords. Il dit avoir commis les sept péchés capitaux, la liste en est donnée de manière assez précise, avoir transgressé les dix commandements. Nous sommes en pleine orthodoxie. La folie réside dans le fait qu'il se croit déjà en enfer. Mais c'est une folie prophétique, puisque cet enfer, où règne Néron et où le Prince des Ténèbres est un gentilhomme, pourrait bien être l'enfer véritable. La folie se met donc au service de l'orthodoxie, elle se fait prédication.

Et pourtant on ne peut pas en rester là. Peu importe que la folie d'Edgar soit feinte. Ce sont des textes que nous étudions, et un énoncé a toujours une signification intrinsèque, indépendante de l'énonciation ; une folie théâtrale est toujours simulée, si ce n'est pas le personnage, c'est par l'auteur. Seulement, la folie fausse ou vraie, n'est pas uniquement une affaire de discours, même en littérature, même sur la scène, très oratoire, très poétique, très philosophique, du théâtre shakespearien. La folie est d'abord une maladie, qui fait souffrir ses victimes. Les fous sont malheureux, ou bien, comme tout est réversible en ce bas monde, le malheur est peut-être une forme de folie. Si Edgar alias Tom de Bedlam se croit en Enfer, ou fait semblant de s'y croire, c'est aussi parce qu'il souffre réellement, du froid, de la faim, de la misère morale et physique. Sa folie est une nudité existentielle que symbolise sa nudité physique. Il subit sans intermédiaire l'agression de tout ce qui est visqueux, gluant, rampant, répugnant, de toute une nature en proie au delirium tremens. Quant à Lear, il décrit lui-même sa démence en termes somatiques. Il a le crâne coupé jusqu'au cerveau, il a la sensation physique de son vertige intérieur, il décrit sa fureur morbide en termes savants, et latins, c'est-à-dire dans un vocabulaire qui signale son appartenance à la conception médicale de l'homme : hysterica passio. Le texte de 1623 porte historica passio, "passion historique», ce qui ne manque pas de cocasserie, mais les éditeurs ont évidemment raison d'adopter la correction proposée dès le quatrième Folio. Tout ceci dénote un certain réductionnisme positiviste de la folie, comme maladie résultant d'un enchaînement de causes, et qui se soigne, notamment par la musicothérapie. Mais la musique évoque par contrecoup la tempête, et pour rendre un bref hommage au grand George Wilson Knight qui nous a quittés cette année même, rappelons que la folie reflète aussi la grande tragédie de l'existence. En conclusion, ne tombons pas dans la passion historique, qui, sous couleur 
de retracer l'histoire de la passion donneraità la folie un visage séduisant. N'envions pas Constance, ni Lady Macbeth. N'envions pas ceux qui souffrent, même si cette souffrance ressemble à une ivresse. Et l'écriture de la folie n'est elle-même au mieux que, selon la formule du poète chinois que traduisit Kans Bethge et que Gustav Malher mit en musique, Das Trinklied vom Jammer der Erde, la chanson à boire de la douleur de la Terre. 